\title{
A family of 2-arc transitive pentagraphs with unbounded valency
}

go back

full screen

close

quit

\author{
Andries E. Brouwer
}

\author{
Johannes Huizinga
}

\section{Abstract}

We construct polygonal graphs on the points of a generalized polygon in general position with respect to a polarity.

Keywords : pentagraphs, generalized polygon, polarity

MSC 2000: 05E18, 20B25, 51E12

\section{Polygonal graphs}

Let $(X, L, I)$ be a generalized $n$-gon with polarity $\sigma$. Let $Z$ be the set of points in general position with respect to $\sigma$, i.e., $Z=\left\{x \in X \mid d\left(x, x^{\sigma}\right) \geq n-1\right\}$, with distances measured in the point-line incidence graph $\Sigma$ of $(X, L, I)$. (Thus, if $n$ is even then $d\left(x, x^{\sigma}\right)=n-1$ and if $n$ is odd then $d\left(x, x^{\sigma}\right)=n$ for $x \in Z$.) Define a graph $\Gamma$ with vertex set $Z$ by letting distinct vertices $x, y \in Z$ be adjacent (notation $x \sim y$ ) when $x I y^{\sigma}$.

Theorem 1.1. If $n$ is odd, then $\Gamma$ has girth $g \geq n$ and each edge is contained in a unique $n$-gon. If $n$ is even, then $\Gamma$ has girth $g \geq n+1$ and each 2-path is contained in a unique $(n+1)$-gon.

Proof. Let us first collect information about the vertex set $Z$.

Step 1. If $x_{0} I x_{1}^{\sigma} I x_{2} I \ldots I x_{l-1} I x_{0}^{\sigma} I x_{1} I \ldots I x_{l-1}^{\sigma} I x_{0}$ is a self-polar 2l-circuit in $\Sigma$, and $l \leq n+1$, then $x_{i} \in Z(0 \leq i \leq l-1)$.

(Indeed, if $d_{\Sigma}\left(x_{i}, x_{i}^{\sigma}\right)=m$, then we find an $(m+l)$-circuit in $\Sigma$, so that $m+l \geq 2 n$.) 



\section{Pentagraphs}

Now let us specialize to the finite case $n=4$, i.e., let $(X, L, I)$ be a generalized quadrangle of order $q$ with a polarity $\sigma$. Then $2 q$ is a square, cf. Payne [4]. Examples exist when $q$ is an odd power of 2, cf. Tits [9]. We define the graph $\Gamma$ as before. As we shall see, $\Gamma$ is a pentagraph, that is, any 2-path in $\Gamma$ is contained in a unique pentagon. (For this concept, and other examples, and some theory, see Perkel [5, 6, 7, 8] and Ivanov [3].)

full screen

close

Theorem 2.1. $\Gamma$ is a pentagraph of valency $q$ on $q^{3}+q$ vertices, and has distance distribution diagram

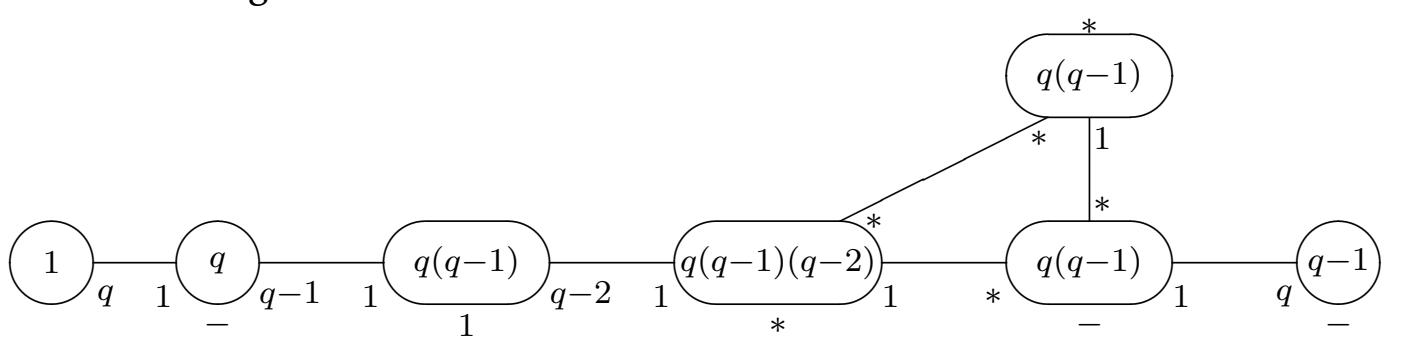

Proof. Recall that a point or line is called absolute (for $\sigma$ ) if it is incident with its image (under $\sigma$ ). We shall use $\sim$ for adjacency in $\Gamma$, and $\perp$ for collinearity in $(X, L)$.

Step 1. Each line contains a unique absolute point, and, dually, each point is on a unique absolute line.

(Indeed, if $x$ is absolute, then $x^{\sigma}$ is the only absolute line on $x$, and if $x$ is not absolute then the unique line on $x$ meeting $x^{\sigma}$ is the only absolute line on $x$.)

Step 2. The set $A$ of absolute points under $\sigma$ is an ovoid in $(X, L)$. The graph $\Gamma$ has $v=q\left(q^{2}+1\right)$ vertices.

(Indeed, each $l \in L$ meets $A$ in a unique point. It follows that $|A|=q^{2}+1$. But $|X|=(q+1)\left(q^{2}+1\right)$.)

Step 3. $\Gamma$ is regular of valency $q$, and does not contain triangles. Adjacent vertices are non-collinear.

(Indeed, the neighbours of $x$ are the $q$ nonabsolute points of $x^{\sigma}$.)

Step 4. $\Gamma$ does not have quadrangles, and any two vertices at distance 2 determine a unique pentagon. Two vertices have distance 2 if and only if they are collinear and the line joining them is non-absolute. 


[5] M. Perkel, Bounding the valency of polygonal graphs with odd girth, Canad. J. Math. 31 (1979), 1307-1321.

[6] A characterization of PSL(2,31) and its geometry, Canad. J. Math. 32 (1980), 155-164.

[7] 9 (1980), 291-298.

[8] - Near-polygonal graphs, Ars Comb. 26A (1988), 149-170.

[9] J. Tits, Ovoides et groupes de Suzuki, Arch. Math. 13 (1962), 187-198.

Andries E. Brouwer

e-mail: aeb@cwi.nl

Johannes Huizinga

e-mail: J.Huizinga@dordrecht.nl 